Image of the Month

\title{
Isolated hypoganglionosis in young man with autism
}

\author{
T. Gabbani ${ }^{\mathrm{a}, *}$, M. Marsico $^{\mathrm{b}}$, M. Marocchi $^{\mathrm{c}}$, M.R. Biagini $^{\mathrm{a}}$ \\ a Oncology Department, Clinical Gastroenterology, AOU Careggi, Florence University Hospital, Florence, Italy \\ ${ }^{\mathrm{b}}$ Gastroenterology and Digestive Endoscopy Unit, Bellaria-Maggiore Hospital, AUSL, Bologna, Italy \\ c Division of Gastroenterology, AOU Modena University Hospital, Modena, Italy
}

A 30-year-old autistic man, presented recurrent sub-occlusive episodes since five years. The CT-scan suggested a bowel obstruction due to volvulus of sigmoid colon with a marked gaseous distention of the right colon inducing liver dislocation on the left and vena cava compression (Fig. 1). A colonoscopy was performed to derotate the volvulus. However, for the appearance of megacolon up to a diameter of $16 \mathrm{~cm}$, a total colectomy was performed. Histological examination showed a framework of intestinal hypoganglionosis (Fig. 2).

Isolated hypoganglionosis $(\mathrm{IH})$ is a rare variant of intestinal dysganglionosis (5\%), usually discovered in infancy or childhood.

This condition shows a markedly decreased number and size of myenteric ganglia, surrounded by CD3 and CD4-positive T lymphocytes, with absent or low acetylcholinesterase activity in the lamina propria as well as hypertrophy of muscularis mucosae and circularmuscle. A deficient expression of c-Kit positive interstitial cells of Cajal and a reduced expression of NCAM-positive nerve fibers were described. Furthermore, the presence of antineuronal nuclear protein antibodies of the ANNA-1 (anti-Hu) type was described in absence of paraneoplastic sensory neuropathy [1].

IH disease usually present severe enterocolitis or chronic constipation and recurrent pseudo-obstruction. Volvulus as first presentation is rare. No association with autism is reported. The diagnosis of IH can only be established by histopathological staining of full-thickness bowel specimens.

\section{Ethical standards statement}

The written consent to the publication of the patient's personal and clinical data was provided to the authors by the legal tutor of the patient.

\section{Reference}

[1] Do MY, Myung SJ, Park HJ, et al. Novel classification and pathogenetic analysis of hypoganglionosis and adult-onset Hirschsprung's disease. Digestive Diseases and Sciences 2011;56:1818-27.

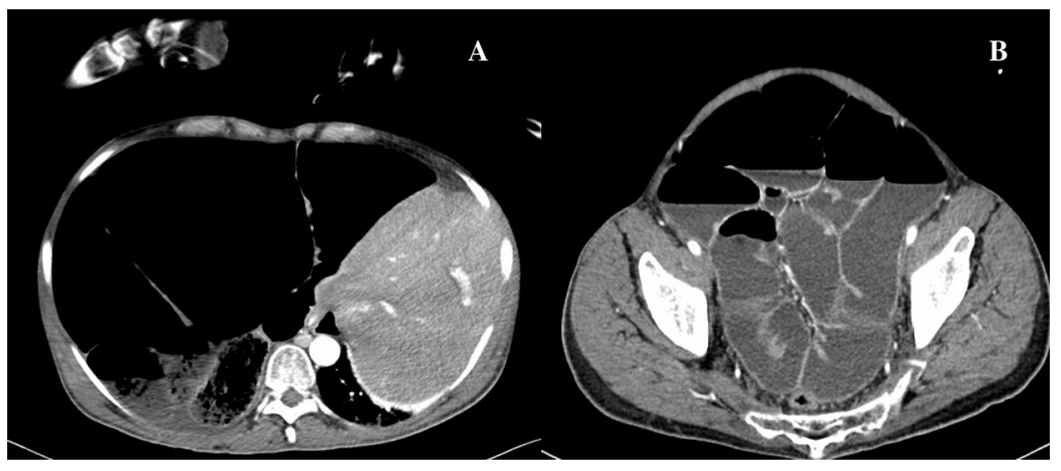

Fig. 1.

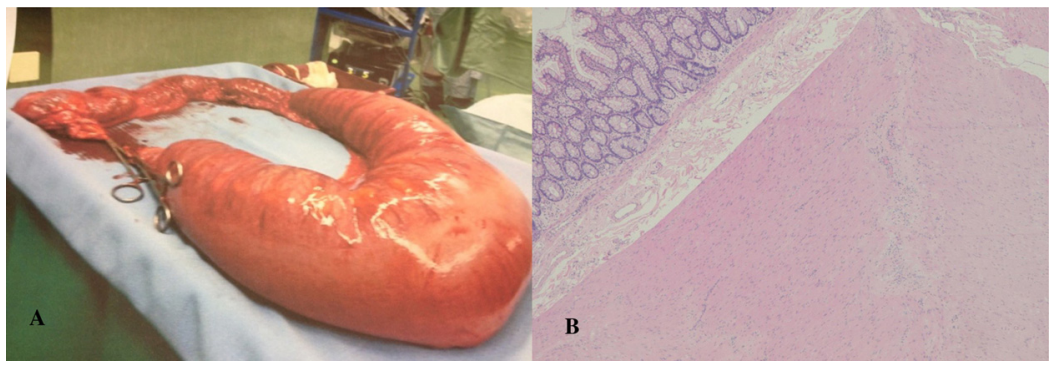

Fig. 2.

\footnotetext{
* Corresponding author.

E-mail address: tom.gabbani@gmail.com (T. Gabbani).
} 